\title{
High expression of eIF4A2 is associated with a poor prognosis in esophageal squamous cell carcinoma
}

\author{
SHANSHAN LYU ${ }^{1 *}$, JIABIN LU $^{2,3 *}$, WENDAN CHEN $^{1}$, WEIYE HUANG ${ }^{1}$, \\ HAOQI HUANG ${ }^{1}$, SHAOYAN XI ${ }^{2,3}$ and SHUMEI YAN ${ }^{2,3}$ \\ ${ }^{1}$ Department of Pathology, Guangdong Provincial People's Hospital/Guangdong Academy of Medical Sciences, \\ Guangzhou, Guangdong 510080; ${ }^{2}$ State Key Laboratory of Oncology in South China, \\ Collaborative Innovation Center for Cancer Medicine; \\ ${ }^{3}$ Department of Pathology, Sun Yat-Sen University Cancer Centre, Guangzhou, Guangdong 510060, P.R. China
}

Received April 12, 2020; Accepted July 27, 2020

DOI: $10.3892 / \mathrm{ol} .2020 .12038$

\begin{abstract}
Eukaryotic initiation factor 4A-II (eIF4A2) is an ATP-dependent RNA helicase involved in mRNA translation. Abnormal expression of eIF4A 2 has been reported as a prognostic factor in different types of cancer. However, little is known regarding the function of eIF4A2 in esophageal squamous cell carcinoma (ESCC). In the present study, 253 samples were collected from patients diagnosed with ESCC, and the expression of eIF4A2 was detected by immunohistochemical staining. The clinicopathological and prognostic significance of eIF4A2 expression in ESCC were then statistically analyzed. The results demonstrated that eIF4A2 was specifically localized to the cytoplasm. Kaplan-Meier analysis also revealed that eIF4A2 expression was associated with the clinical prognosis of patients with ESCC. The median disease-free and overall survival times were 40 and 48 months for patients with low eIF4A2 expression, compared with 16 and 25 months in the high eIF4A2 expression group, respectively. In conclusion, high expression levels of eIF4A2 are associated with a poor prognosis and may be used as a potential prognostic indicator in patients with ESCC.
\end{abstract}

\section{Introduction}

Esophageal cancer (EC) is one of the most common types of cancer worldwide and is the sixth most common type of malignancy (1). The incidence of EC has markedly increased

Correspondence to: Dr Shumei Yan or Dr Shaoyan Xi, State Key Laboratory of Oncology in South China, Collaborative Innovation Center for Cancer Medicine, 651 Dong-Feng Road East, Guangzhou, Guangdong 510060, P.R. China

E-mail: yanshm@sysucc.org.cn

E-mail: xishy@sysucc.org.cn

*Contributed equally

Key words: eukaryotic initiation factor 4A-II, immunohistochemistry, esophageal squamous cell carcinoma, prognosis in recent decades. For example, $\sim 572,034$ new case diagnoses and 508,585 mortalities were reported in 2018 alone (1). EC is histologically categorized into two subtypes, esophageal squamous cell carcinoma (ESCC) and esophageal adenocarcinoma (EA), and the incidence varies by histological type. In addition, the geographical location also appears to influence the incidence rate of EC. For example, previous studies have suggested that regions, including China, the Middle East and Southern Africa exhibit high rates of ESCC (2-4). As there are initially no detectable symptoms, the majority of patients are diagnosed at the advanced stages of EC, resulting in a poor overall prognosis. At present, available treatments for EC include surgery, chemotherapy and radiotherapy. Unlike other malignancies, including lung and breast cancer, there are few molecular markers available for ESCC which provide guidance for treatment options and facilitate prognostic predictions (5). Therefore, the identification of novel biomarkers for ESCC is of considerable importance.

As a frequent characteristic of human cancer, the dysregulation of mRNA translation may result in tumor growth, metastasis, angiogenesis and escape from immune eradication $(6,7)$. The initiation phase is the rate-limiting and most tightly regulated step in translational control. Existing studies have established an associated between various types of eukaryotic initiation factors (EIFs) and the genesis and prognosis of different cancer types (8-13). In eukaryotes, eukaryotic initiation factor $4 \mathrm{~A}$ (eIF4A) is essential for translation initiation. It is a canonical DEAD-box helicase, responsible for unwinding the extensive secondary structures of the 5' untranslated regions of mRNAs during 40S ribosomal subunit scanning (14). There are three eIF4A isoforms in mammals, i.e. eIF4A1-3 (15). As a nuclear protein and component of the exon junction complex (EJC), eIF4A3 is distinct from the other isoforms, and is essential in anchoring the EJC to the RNA molecule (16). Secondly, eIF4A1 and eIF4A2 share $91 \%$ amino acid sequence homology and are considered to be functionally indistinguishable (17). However, recent studies have reported that unlike eIF4A1, eIF4A2 interacts with the CCR4-NOT transcription complex and is involved in the inhibition of miRNA-mediated translation (18). As such, the upregulation of eIF4A1 is reportedly associated with a 
diverse range of malignancies, including non-small cell lung cancer (NSCLC), endometrial carcinoma, cervical cancer, breast cancer, malignant peripheral nerve sheath tumors and pancreatic ductal adenocarcinoma (PDA) (19-24). Several relevant studies have also demonstrated that the expression of eIF4A 2 is positively correlated with the prognosis of NSCLC and breast cancer $(25,26)$. However, a recent study revealed that high eIF4A2 expression in colorectal cancer (CRC) was associated with a poor survival rate. Additionally, the results of cellular and animal experiments have confirmed the functions of eIF4A2 in promoting CRC metastasis and oxaliplatin resistance (27). Such discrepancies may be attributed to the cancer type or the diversity of oncogene-stimulated signaling networks. As a result, the association between eIF4A2 expression and any specific type of cancer demands independent research and cautious investigation.

In the present study, 253 ESCC patient samples were collected and the expression of eIF4A2 was detected by immunohistochemical (IHC) staining. The function of eIF4A2 in the prognosis of ESCC was then ascertained.

\section{Materials and methods}

Patient and tissue specimens. The present study was approved by the Medical Ethics Committee of Sun Yat-Sen University Cancer Center (Guangzhou, China), and all enrolled patients provided written informed consent. A total of 253 patient specimens from 186 males and 67 females; the median patient age was 58 years (age range, $32-80$ years) were collected from post-operative patients with ESCC, the validity of which were confirmed by pathological review following IHC staining. All 253 patients were observed at Sun Yat-Sen University Cancer Center (Guangzhou, China) between January 2000 and December 2007, during which they were clinically and histologically diagnosed with ESCC. The histological grade and clinical stage of the tumors were recorded according to the 8th edition of the Tumor-Node-Metastasis (TNM) classification of the International Union Against Cancer (2018) (28). A patient was selected as a qualifiable subject if the following five requirements were met: i) The patient was diagnosed with histologically confirmed primary ESCC, but had not received any previous treatment elsewhere; ii) the patient had no family history of cancer; iii) the patient had undergone radical surgery with lymphadenectomy (limited or extended); iv) the patient had not received neoadjuvant or adjuvant treatments; and $v$ ) the clinical information and follow-up data of the patient were documented. As a result, 253 patients constituted the complete set of specimens, and their clinical data were obtained from hospital records. The patients were followed up in 2019 to ascertain the latest disease status.

A tissue microarray was constructed according to previously specified methods (29). Samples from the 253 patient specimens were fixed in formalin for 24 hours at room temperature and then embedded in paraffin. The paraffin blocks were sliced into 3- $\mu \mathrm{m}$ sections. The sections were then reviewed by a senior pathologist and representative tumor regions were defined by hematoxylin and eosin staining. Next, two targeted core samples from each tissue specimen were obtained using a tissue array instrument (MiniCore ${ }^{\circledR}$ instruments; ALPHELYS). Tissue cylinders with a diameter of $10 \mathrm{~mm}$ were punched and arrayed in a recipient paraffin block. Sections of the tissue array were then cut and arranged on glass slides.

IHC staining and assessment. Following paraffin embedding, the tissue sections were dried in incubator at $60^{\circ} \mathrm{C}$ for $2 \mathrm{~h}$, then deparaffinized in xylene and rehydrated in a descending graded alcohol series (100, 95, 90, 85 and 75\%), and then incubated in $0.3 \%$ hydrogen peroxide for $15 \mathrm{~min}$ to block endogenous peroxidase activity. Antigen retrieval was conducted by heating in EDTA buffer ( $\mathrm{pH} \mathrm{8.0)}$ for $3 \mathrm{~min}$ in a pressure cooker. The sections were blocked for $10 \mathrm{~min}$ with $10 \%$ normal goat serum (cat. no. ab7481; Abcam) at room temperature, and then incubated with a polyclonal antibody against eIF4A2 (dilution, 1:1,000; cat. no. ab31218; Abcam) for $12 \mathrm{~h}$ in a moist chamber $\left(4^{\circ} \mathrm{C}\right)$. Control samples were incubated in blocking solutions without the primary antibody. The slides were then incubated with horseradish peroxidase for $30 \mathrm{~min}$ and visualized using 3,30-diaminobenzidine solution. Mayer's hematoxylin was applied as a counterstain at room temperature for $1 \mathrm{~min}$. Negative control staining was performed by replacing the primary antibody with normal murine immunoglobulin G (1:200; cat. no. A7028; Beyotime) for $12 \mathrm{~h}$ in a moist chamber at $4^{\circ} \mathrm{C}$. eIF4A2-positive slides were used as positive controls, and the internal negative controls consisted of normal squamous esophageal mucosal samples from cancer-free patients.

Next, two independent pathologists (Lyu and Yan) blinded to the clinicopathological data evaluated eIF4A2 expression in the tissue specimens with a light microscope (magnifications, $\mathrm{x} 100$ and $\mathrm{x} 200$ ). The evaluation approach followed two scoring criteria: i) The positive cell proportion score was assigned according to the percentage of positive tumor cells: 0 (0\%), 1 (1-10\%), 2 (11-50\%), 3 (51-70\%) and 4 (71-100\%); and ii) the staining intensity score was graded in accordance with the signal intensity: 0 (no signal), 1 (weak), 2 (medium) and 3 (strong). The immunoreactivity score (IRS) for eIF4A2 expression was calculated by multiplying the positive cell proportion and the staining intensity scores (range, $0-12$ ). The specimens were re-evaluated if the scores submitted by the two pathologists displayed discrepancy. In addition, eIF4A2 expression data were downloaded from UALCAN (http:// ualcan.path.uab.edu/index.html), an interactive web portal for the in-depth analysis of The Cancer Genome Atlas (TCGA) gene expression data.

Selection of cut-off values. The survival receiver operating characteristic (ROC) package in $\mathrm{R}$ ( $\mathrm{R}$ software version 3.0.1 and the survival ROC package; R Foundation for Statistical Computing; www.R-project.org) was used to determine the IRS cut-off values. The sensitivity and specificity for each outcome were plotted and ROC curves were generated. The score was selected as the cut-off value which was closest to the point with both maximum sensitivity and specificity. For eIF4A2 expression, the cut-off value for the staining intensity score (0-3), proportion score (0-4) and IRS (0-12) were assessed separately. Clinicopathological features, including age (68 years old or $>50$ years old), sex (male or female), histological grade (1-2 or 3), tumor status (T1, T2, T3 or T4), nodal status (N0 or N1) and TNM stage (1-2 or 3 ) were also evaluated. 
Statistical analysis. Survival analysis was performed using SPSS version 25.0 (IBM Corp.). Pearson's $\chi^{2}$ test was used to determine the association between eIF4A2 expression and patient clinicopathological features. Disease-free survival (DFS) was defined as the time between surgery and regional relapse or the development of distant metastasis. Overall survival (OS) was defined as the time between surgery and death. The Kaplan-Meier estimator was used to assess DFS and OS, the results of which were further compared using the log-rank test. Multivariate survival analysis was performed using the Cox regression model for all variables that were identified as significant during univariate analysis. For all analyses, $\mathrm{P}<0.05$ (two-sided) was considered to indicate a statistically significant difference.

\section{Results}

Demographics. Of the study population, 59 patients were diagnosed with well-differentiated squamous cell carcinoma (SCC), 163 patients presented with moderately-differentiated SCC and 31 with poorly-differentiated SCC. The number of patients confirmed to have $\mathrm{T} 1, \mathrm{~T} 2, \mathrm{~T} 3$ and $\mathrm{T} 4$ disease was 6,61 , 183 and 3, respectively, and 118 of the 253 patients exhibited nodal metastases. With regards to TNM staging, 156 patients were diagnosed with stage 1 and 2 disease, and 97 patients presented with stage 3 disease. The mortality rate across all patients was $52.6 \%$ (133 out of 253), and the median OS and DFS times were 40 (4-115) and 38 (1-115) months, respectively.

IHC detection of eIF4A2 expression in ESCC. The results of the present study demonstrated that eIF4A2 expression is localized to the cytoplasm of ESCC cells. eIF4A2 was also revealed to be more highly expressed in esophageal carcinoma tissues than in the normal control samples, displaying a similar expression trend to that determined by TCGA database analysis, though not statistically significant (Figs. 1 and 2). In the present study, samples with a signal intensity score of 3 (strong) were considered to exhibit high eIF4A2 expression, while those with a score of 1 (weak) or 2 (medium) were considered to exhibit low expression intensity. High eIF4A2 expression was detected in 53 patients, while the remaining 200 samples presented with low eIF4A2 expression. None of the samples scored 0 (Fig. 2). The cut-off value for the proportion score $(0-4)$ was 1 , indicating that samples with a score $\leq 1$ were considered to exhibit a low proportion of eIF4A2 expression, and those with a score of $>1$ were regarded to express a high proportion of eIF4A2. There were 10 patients with a low, and 243 patients with a high proportion of eIF4A2 expression. For the IRS (0-12), ESCC samples with a score $>9$ were defined as highly eIF4A2-immunoreactive. Therefore, in the present study, 214 patients possessed high eIF4A2 immunoreactivity and the remaining 39 patients presented with low immunoreactivity. However, Kaplan-Meier analysis revealed that only the intensity score was significantly associated with DFS and OS, hence a high eIF4A2 intensity was defined as high eIF4A2 expression, and low intensity samples were defined by low eIF4A2 expression (Table I).

Association between eIF4A2 expression and the clinicopathological characteristics of patients with ESCC. There were

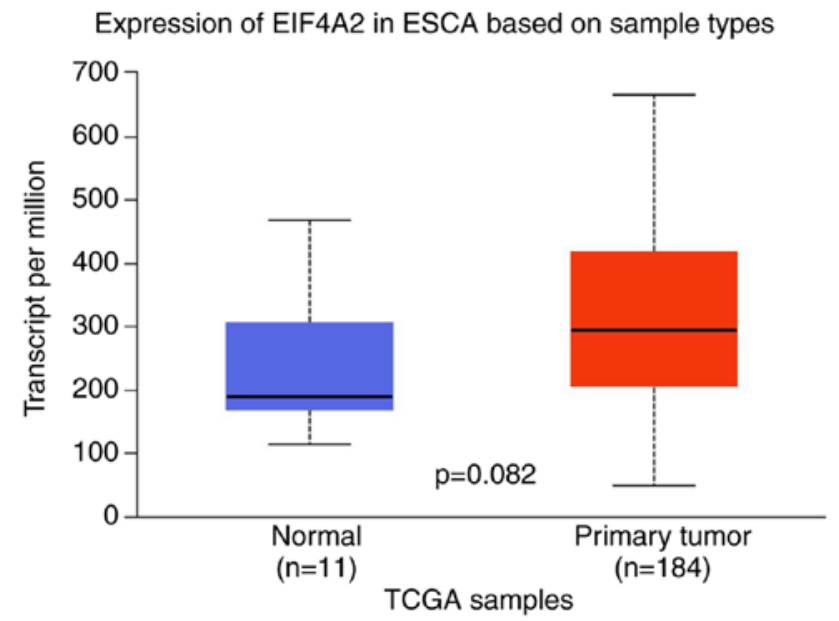

Figure 1. Expression of eIF4A2 in TCGA database. A total of 11 normal cases [median, 188.31 (115.03-467.41)] and 184 patients with ESCA [median, 295.32 (50.15-666.01)] from TCGA samples were assessed. $\mathrm{P}=0.082$ (Student's $t$-test). eIF4A2, eukaryotic initiation factor 4A-II; TCGA, The Cancer Genome Atlas; ESCA, esophageal carcinoma.

no statistically significant associations between the general clinicopathological characteristics of the 253 patients and eIF4A2 expression (Table II).

Association between eIF4A2 expression and the prognosis of patients with ESCC. According to the results of Kaplan-Meier analysis, specific clinicopathological characteristics, including age, histological grade, tumor, nodal and TNM staging were revealed to be significantly associated with DFS and OS time $(\mathrm{P}=0.002$; Fig. $3 \mathrm{C}$ and $\mathrm{D})$; the cut-off value for age was set at 68 years. eIF4A2 expression was also revealed to be associated with the prognosis of ESCC. The median DFS time was 40 months for patients with low eIF4A2 expression and 16 months for those with high eIF4A2 expression $(\mathrm{P}<0.001$; Fig. 3A). In addition, the median OS time was 48 months for patient with low eIF4A2 expression, but 25 months for those with high eIF4A2 expression ( $\mathrm{P}<0.001$; Fig. 3B). When patient specimens were stratified into $\mathrm{N}$ stage subgroups, the association between eIF4A2 expression and DFS or OS became more significant. In the N0 subgroup, the median DFS and OS times of patients with low eIF4A2 expression were 62 and 65 months, respectively, while the median DFS and OS times of those with high eIF4A2 expression were both 40 months (both $\mathrm{P}=0.002$ ). In the N1 subgroup, the median DFS and OS times of patients with low eIF4A2 expression were 19 and 27 months, respectively, while the median DFS and OS times of those with high eIF4A2 expression were 10 and 15 months, respectively (both $\mathrm{P}=0.002)$. These data indicate that high expression levels of eIF4A2 are associated with a poor prognosis in patients with ESCC (Fig. 3).

Univariate analysis was performed using the Cox proportional hazards model to assess the importance of multiple factors on the survival times of patients with ESCC. The results indicated that age, histological grade, tumor and nodal status, TNM stage and eIF4A2 expression are significantly associated with DFS and OS time (Table I). Multivariate analysis was then applied to investigate these identified parameters. The results verified that only eIF4A 2 expression and age 
Table I. Univariate Cox regression analysis of DFS and OS in patients with esophageal squamous cell carcinoma.

\begin{tabular}{|c|c|c|c|c|}
\hline \multirow[b]{2}{*}{ Variable } & \multicolumn{2}{|c|}{ DFS } & \multicolumn{2}{|c|}{ OS } \\
\hline & HR $(95 \%$ CI) & P-value & $\mathrm{HR}(95 \% \mathrm{CI})$ & P-value \\
\hline \multicolumn{5}{|c|}{ Ages, years } \\
\hline$>68$ & 2.053 & 0.002 & 1.929 & 0.004 \\
\hline$\leq 68$ & $(1.297-3.250)$ & & $(1.218-3.054)$ & \\
\hline \multicolumn{5}{|l|}{ Sex } \\
\hline Male & 1.202 & 0.359 & 1.240 & 0.284 \\
\hline Female & $(0.811-1.782)$ & & $(0.837-1.839)$ & \\
\hline \multicolumn{5}{|c|}{ Histological grade } \\
\hline $1-2$ & 1.791 & 0.012 & 1.699 & 0.022 \\
\hline 3 & $(1.132-2.883)$ & & $(1.074-2.689)$ & \\
\hline \multicolumn{5}{|c|}{ Tumor status } \\
\hline T1 & 1.456 & 0.047 & 1.523 & 0.026 \\
\hline $\mathrm{T} 2$ & $(1.004-2.114)$ & & $(1.050-2.211)$ & \\
\hline \multicolumn{5}{|l|}{ T3 } \\
\hline \multicolumn{5}{|l|}{$\mathrm{T} 4$} \\
\hline \multicolumn{5}{|c|}{ Nodal status } \\
\hline NO & 3.306 & $<0.001$ & 3.452 & $<0.001$ \\
\hline N1 & $(2.299-4.754)$ & & $(2.399-4.976)$ & \\
\hline \multicolumn{5}{|c|}{ TNM stage } \\
\hline $1-2$ & 3.494 & $<0.001$ & 3.751 & $<0.001$ \\
\hline 3 & $(2.456-4.972)$ & & $(2.631-5.349)$ & \\
\hline \multicolumn{5}{|c|}{ eIF4A2 expression } \\
\hline Low & 1.948 & $<0.001$ & 1.956 & $<0.001$ \\
\hline High & $(1.347-2.818)$ & & $(1.352-2.829)$ & \\
\hline \multicolumn{5}{|c|}{ Proportion of eIF4A2 expression } \\
\hline Low & 0.520 & 0.086 & 0.576 & 0.151 \\
\hline High & $(0.243-1.113)$ & & $(0.269-1.234)$ & \\
\hline \multicolumn{5}{|c|}{ IRS for eIF4A2 expression } \\
\hline Low & 1.441 & 0.089 & 1.473 & \\
\hline High & $(0.944-2.189)$ & & $(0.965-2.248)$ & 0.071 \\
\hline
\end{tabular}

DFS, disease-free survival; OS, overall survival; HR, hazard ratio; CI, confidence interval; TNM, Tumor-Node-Metastasis; eIF4A2, eukaryotic initiation factor 4A-II; IRS, immunoreactivity score.

were independent prognostic factors for DFS and OS time in ESCC (Table III).

\section{Discussion}

In the present study, the expression of eIF4A2 in ESCC samples was investigated by IHC staining. TCGA database analysis revealed that eIF4A2 is more highly expressed in tumor tissues than in normal control samples, and that the highest amplification frequency of eIF4A2 is detected in ESCC (Figs. 1 and 2). Biologically identified as an ATP-dependent RNA helicase, eIF4A2 belongs to the eukaryotic initiation factor (eIF) family, which is essential for translation initiation (30). Existing findings have established various associations between the eIFs and carcinogenesis, as well as patient prognosis $(11,13,24)$. For example, the upregulation of eIF4E functions as an effective indicator for $30 \%$ of all cancer cases tested, and the phosphorylation of eIF4E promotes cellular proliferation and inhibits apoptosis (12). eIF4A2 was the focus of the present study, and is a subunit of eIF4A that is associated with the oncogenic translation of PDA (24). eIF4A is a subunit of eIF4F that also comprises eIF4E (a cap binding protein) and eIF4G (a regulatory scaffold protein). The eIF4F family serves an important role in the translation process. eIF4A comprises three subunits, eIF4A1, eIF4A2 and eIF4A3. Within the eIF4A family, the amino acid sequences of eIF4A1 and eIF4A2 are homologues and are highly conserved $(8,17)$, and dysregulation of either of these molecules is associated with a diverse range of malignancies $(20,22,25,27)$. In the present study, the expression rate of eIF4A 2 in the ESCC cohort was $100 \%$ (253 out of 253). Additionally, 20.9\% (53 out of 
Table II. Association between specific clinicopathological characteristics and eIF4A2 expression in patients with esophageal squamous cell carcinoma.

eIF4A2 expression

\begin{tabular}{|c|c|c|c|c|}
\hline & & & & \\
\hline Variable & Cases, $\mathrm{n}$ & Low $(n=200)$ & High $(n=53)$ & P-value \\
\hline Ages, years & & & & 0.353 \\
\hline$>68$ & 29 & 21 & 8 & \\
\hline$\leq 68$ & 224 & 179 & 45 & \\
\hline Sex & & & & 0.301 \\
\hline Male & 186 & 150 & 36 & \\
\hline Female & 67 & 50 & 17 & \\
\hline Histological grade & & & & 0.071 \\
\hline 1 & 59 & 51 & 8 & \\
\hline 2 & 163 & 127 & 36 & \\
\hline 3 & 31 & 22 & 9 & \\
\hline Tumor status & & & & 0.279 \\
\hline $\mathrm{T} 1$ & 6 & 4 & 2 & \\
\hline $\mathrm{T} 2$ & 61 & 53 & 8 & \\
\hline $\mathrm{T} 3$ & 183 & 141 & 42 & \\
\hline $\mathrm{T} 4$ & 3 & 2 & 1 & \\
\hline Nodal status & & & & 0.482 \\
\hline N0 & 135 & 109 & 26 & \\
\hline $\mathrm{N} 1$ & 118 & 91 & 27 & \\
\hline TNM stage & & & & 0.116 \\
\hline 1 & 9 & 8 & 1 & \\
\hline 2 & 147 & 120 & 27 & \\
\hline 3 & 97 & 72 & 25 & \\
\hline
\end{tabular}

eIF4A2, eukaryotic initiation factor 4A-II; TNM, Tumor-Node-Metastasis.

253) of patient specimens exhibited high eIF4A2 expression. However, no statistically significant correlations were detected between eIF4A2 expression and general clinicopathological characteristics. An existing study demonstrated that eIF4A2 is more highly expressed in lung SCC than in adenocarcinoma, and that eIF4A2 downregulation indicates a poor prognosis in patients with NSCLC (25). The present study had a distinct focus and established that high eIF4A2 expression is associated with a poor patient prognosis in ESCC.

Another aim of the present study was to investigate the association between abnormal eIF4A2 expression and the prognosis of patients with ESCC. High eIF4A2 expression was revealed to be significantly associated with a poor outcome in ESCC, as determined by Kaplan-Meier and log-rank analysis. However, this analytical approach tends to produce false positive results. The log-rank test can more sensitively predict long-term differences, while the Breslow test is more appropriately used to predict short-term differences in ending events. Therefore, when analyzing survival curves with similar data values over a given time frame, the log-rank test is more likely to generate a significant result than the Breslow test. By contrast, for survival curves that differ considerably at the initial time points, but draw closer over time, significant results are more likely to be obtained using the Breslow method. The results of the present study were verified using the Breslow test $(\mathrm{P}<0.001)$, and univariate analysis was performed using the Cox proportional hazards model to correct confounding factors $(\mathrm{P}=0.003)$. Therefore, the final results were concluded to be reliable.

The association between eIF4A2 and clinicopathological characteristics became statistically significant when the patients were classified into different $\mathrm{N}$ stage subgroups, which was also consistent with the survival curve trend shown in TCGA database. eIF4A1 and eIF4A2 share a 91\% homologous amino acid sequence and are considered to be functionally indistinguishable (17). Increased expression of eIF4A1 has been associated with NSCLC metastasis and the poor outcomes of patients with cervical and breast cancer. Knocking down eIF4A2 has also been reported to inhibit sphere formation and experimental metastasis in CRC, and eIF4A2 expression was revealed to be positively correlated with the prognosis of patients with NSCLC and breast cancer $(20,22,27)$. Furthermore, TCGA analysis revealed that high eIF4A2 expression is negatively correlated with the prognosis of those with liver cancer, head and neck cancer, melanoma and prostate cancer. The Bushell lab demonstrated that eIF4A2 contributes toward the repression of miRNA translation initiation via an interaction between DDX6 and the CCR4-NOT complex, 
A

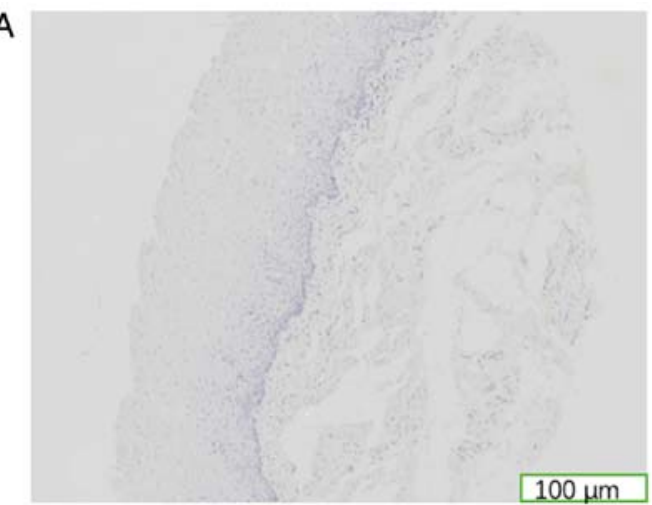

C

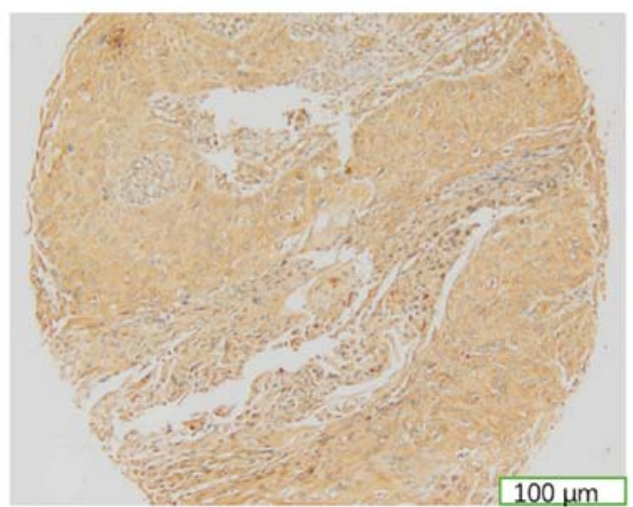

B

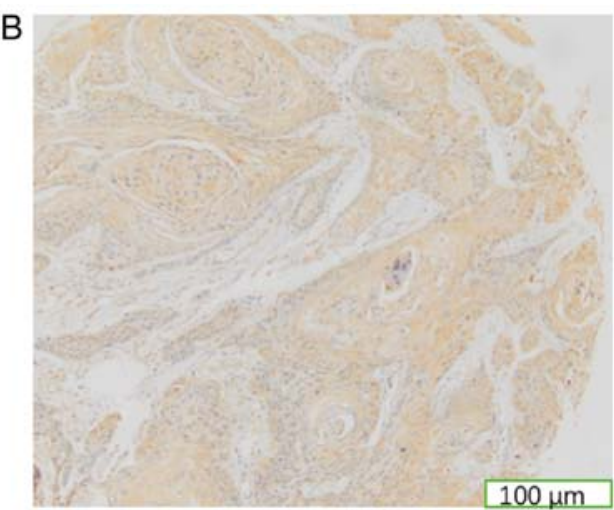

D

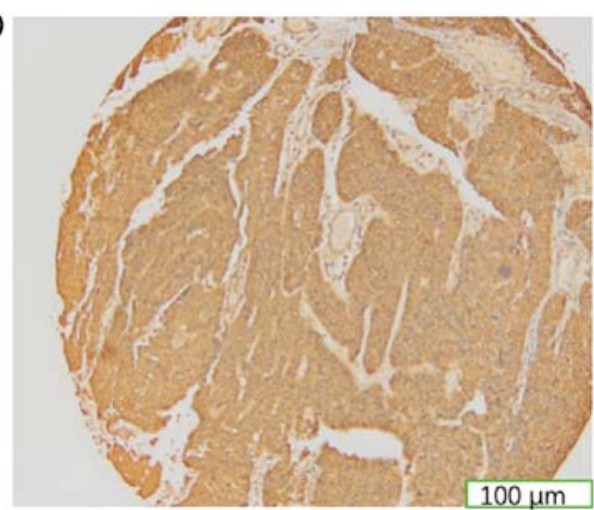

Figure 2. Detection of eIF4A2 in ESCC samples by immunohistochemical staining. (A) eIF4A2 expression in control, normal squamous mucosa tissues of the esophagus. (B) Weak, (C) medium and (D) strong eIF4A2 expression in ESCC tissues. eIF4A2, eukaryotic initiation factor 4A-II; ESCC, esophageal squamous cell carcinoma.
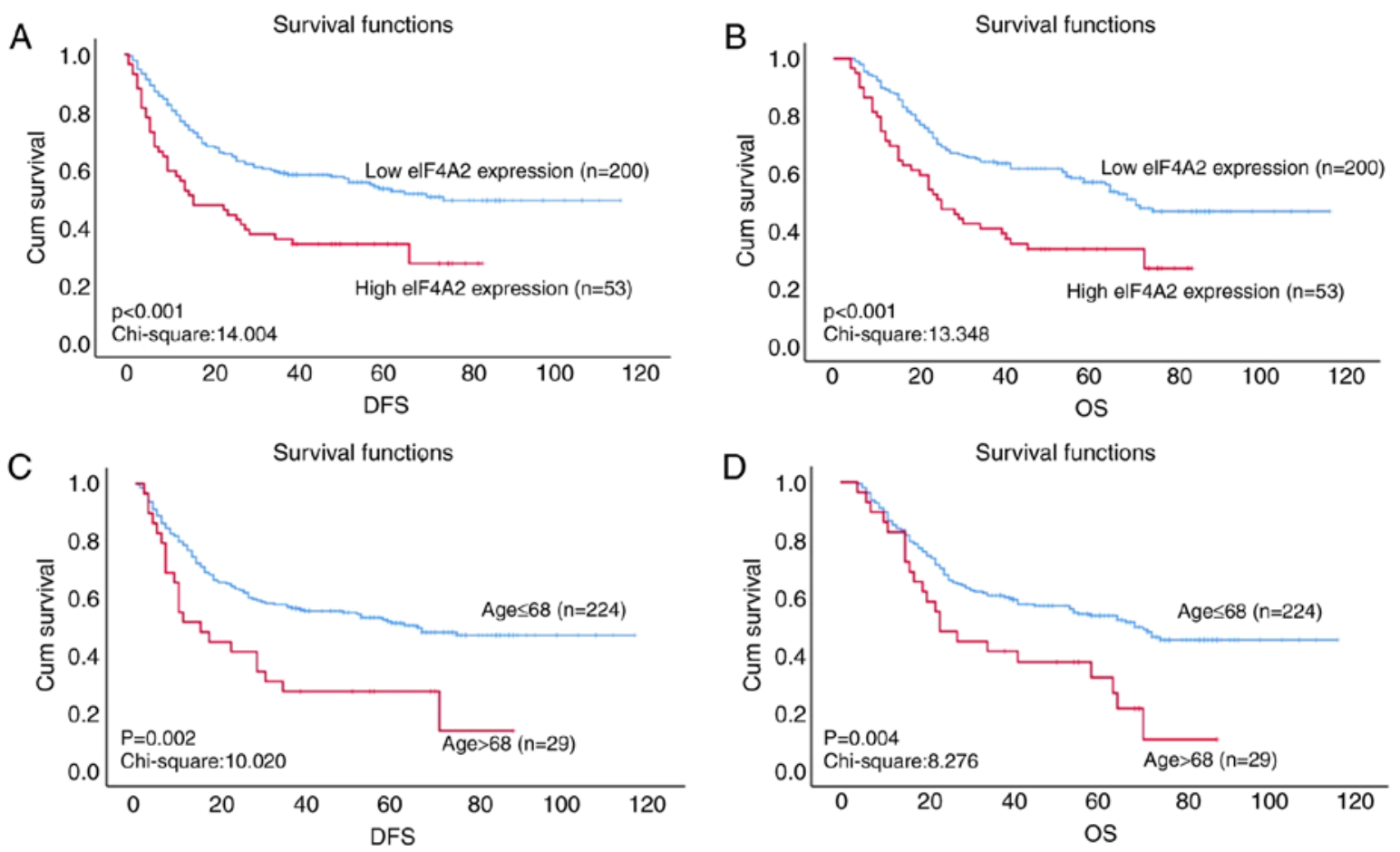

Figure 3. DFS and OS curves of eIF4A2 expression in patients with ESCC. (A) DFS and (B) OS curves for ESCC patients with high and low eIF4A2 expression ( $\mathrm{P}<0.001)$. (C) DFS and (D) OS curves of patients aged $\leq 68$ and $>68$ years old $(\mathrm{P}=0.002$ and $\mathrm{P}=0.004$, respectively). DFS, disease-free survival; OS, overall survival; eIF4A2, eukaryotic initiation factor 4A-II; ESCC, esophageal squamous cell carcinoma.

and that eIF4A2 inhibits the deadenylation activity of CNOT7 subunit, which further results in translational repression (31).
eIF4A2 has also been hypothesized to function through the Myc pathway, though further investigation is required to confirma- 
Table III. Multivariate Cox regression analysis of DFS and OS in patients with esophageal squamous cell carcinoma.

\begin{tabular}{|c|c|c|c|c|}
\hline \multirow[b]{2}{*}{ Variable } & \multicolumn{2}{|c|}{ DFS } & \multicolumn{2}{|c|}{ OS } \\
\hline & HR (95\% CI) & P-value & $\operatorname{HR}(95 \% \mathrm{CI})$ & P-value \\
\hline \multicolumn{5}{|c|}{ Ages, years } \\
\hline$>68$ & 2.0210 & 0.003 & 1.985 & 0.004 \\
\hline$\leq 68$ & $(1.269-3.219)$ & & $(1.246-3.162)$ & \\
\hline \multicolumn{5}{|c|}{ Histological grade } \\
\hline $1-2$ & 1.577 & 0.052 & 1.537 & 0.068 \\
\hline 3 & $(0.995-2.500)$ & & $(0.969-2.437)$ & \\
\hline \multicolumn{5}{|c|}{ Tumor status } \\
\hline $\mathrm{T} 1$ & 1.030 & 0.905 & 1.088 & 0.733 \\
\hline $\mathrm{T} 2$ & $(0.636-1.667)$ & & $(0.670-1.768)$ & \\
\hline \multicolumn{5}{|l|}{ T3 } \\
\hline \multicolumn{5}{|l|}{$\mathrm{T} 4$} \\
\hline \multicolumn{5}{|c|}{ Nodal status } \\
\hline No & 2.063 & 0.06 & 2.128 & 0.054 \\
\hline N1 & $(0.969-4.391)$ & & $(0.989-4.581)$ & \\
\hline \multicolumn{5}{|c|}{ TNM stage } \\
\hline $1-2$ & 1.772 & 0.175 & 1.828 & 0.16 \\
\hline 3 & $(0.775-4.050)$ & & $(0.789-4.239)$ & \\
\hline \multicolumn{5}{|c|}{ eIF4A2 expression } \\
\hline Low & 1.755 & 0.003 & 1.804 & 0.002 \\
\hline High & $(1.210-2.545)$ & & $(1.244-2.618)$ & \\
\hline
\end{tabular}

DFS, disease-free survival; OS, overall survival; HR, hazard ratio; CI, confidence interval; TNM, Tumor-Node-Metastasis; eIF4A2, eukaryotic initiation factor 4A-II.

tion this (27). In the present study, the Cox proportional hazards model identified eIF4A2 expression as an independent prognostic factor for ESCC. However, the molecular mechanisms by which eIF4A2 dysregulation contributes toward the prognosis of ESCC remain unclear. The discrepancy in the correlation between eIF4A2 expression and cancer may be attributed to the nature of the different cancer types, or the diversity of oncogene-stimulated signaling networks.

In the present study, age was also identified as an independent prognostic factor, and was significantly correlated with DFS and OS time. The cut-off value for age was set at 68 years, indicating that patients $>68$ years old with ESCC had poorer outcomes. Previous studies have also identified sex, $\mathrm{T}$ staging and $\mathrm{N}$ staging as independent prognostic indicators of $\operatorname{ESCC}(4,32)$. Due to the limited sample size of the present study, univariate analysis revealed an association between eIF4A2 expression and patient clinicopathological characteristics, including histological grade, tumor, nodal and TNM staging, and survival without characterizing these factors as independent indicators.

In conclusion, there are two major limitations to the present study: i) The molecular mechanisms by which eIF4A2 dysregulation contributes toward the prognosis of ESCC remain unclear, and require further investigation; and ii) the sample size of the study was not sufficient to establish the correlation between eIF4A2 expression and general clinicopathological characteristics in survival analysis. However, these findings demonstrate the association between high eIF4A2 expression and the poor prognosis of patients, as well as the potential of eIF4A2 as an effective prognostic indicator in ESCC.

\section{Acknowledgements}

Not applicable.

\section{Funding}

No funding was received.

\section{Availability of data and materials}

The datasets used and/or analyzed during the current study are available from the corresponding author on reasonable request.

\section{Authors' contributions}

SY and SX are responsible for the study design. SL and JL performed the experiments and draft the manuscript. WC, WH and $\mathrm{HH}$ participated in the data analysis and interpretation. All authors have read and approved the final manuscript.

\section{Ethics approval and consent to participate}

The present study was approved by the Medical Ethics Committee of Sun Yat-Sen University Cancer Center (approval 
no. GZR2018-220) (Guangzhou, China) and all patients provided written informed consent.

\section{Patient consent for publication}

Not applicable.

\section{Competing interests}

The authors declare that they have no competing interests.

\section{References}

1. Bray F, Ferlay J, Soerjomataram I, Siegel RL, Torre LA and Jemal A: Global cancer statistics 2018: GLOBOCAN estimates of incidence and mortality worldwide for 36 cancers in 185 countries. CA Cancer J Clin 68: 394-424, 2018

2. Gholipour C, Shalchi RA and Abbasi M: A histopathological study of esophageal cancer on the western side of the Caspian littoral from 1994 to 2003. Dis Esophagus 21: 322-327, 2008

3. Pickens A and Orringer MB: Geographical distribution and racial disparity in esophageal cancer. Ann Thorac Surg 76 S1367-S1369, 2003

4. Chen MF, Yang YH, Lai CH, Chen PC and Chen WC: Outcome of patients with esophageal cancer: A nationwide analysis Ann Surg Oncol 20: 3023-3030, 2013

5. Smyth EC, Lagergren J, Fitzgerald RC, Lordick F, Shah MA, Lagergren P and Cunningham D: Oesophageal cancer. Nat Rev Dis Primers 3: 17048, 2017

6. Bhat M, Robichaud N, Hulea L, Sonenberg N, Pelletier J and Topisirovic I: Targeting the translation machinery in cancer. Nat Rev Drug Discov 14: 261-278, 2015.

7. Spilka R, Ernst C, Mehta AK and Haybaeck J: Eukaryotic translation initiation factors in cancer development and progression. Cancer Lett 340: 9-21, 2013.

8. Aitken CE and Lorsch JR: A mechanistic overview of translation initiation in eukaryotes. Nat Struct Mol Biol 19: 568-576, 2012.

9. Hinnebusch AG and Lorsch JR: The mechanism of eukaryotic translation initiation: New insights and challenges. Cold Spring Harb Perspect Biol 4: a011544, 2012.

10. Donzé O, Jagus R, Koromilas AE, Hershey JW and Sonenberg N Abrogation of translation initiation factor eIF-2 phosphorylation causes malignant transformation of NIH 3 T3 cells. EMBO J 14 3828-2834, 1995.

11. Shahbazian D, Parsyan A, Petroulakis E, Topisirovic I, Martineau Y, Gibbs BF, Svitkin Y and Sonenberg N: Control of cell survival and proliferation by mammalian eukaryotic initiation factor 4B. Mol Cell Biol 30: 1478-1485, 2010.

12. Bitterman PB and Polunovsky VA: eIF4E-mediated translational control of cancer incidence. Biochim Biophys Acta 1849, 774-780: 2015.

13. Bao Y, Lu Y, Wang X, Feng W, Sun X, Guo H, Tang C, Zhang X, Shi $\mathrm{Q}$ and $\mathrm{Yu} \mathrm{H}$ : Eukaryotic translation initiation factor 5A2 (eIF5A2) regulates chemoresistance in colorectal cancer through epithelial mesenchymal transition. Cancer Cell Int 15: 109, 2015.

14. Wolfe AL, Singh K, Zhong Y, Drewe P, Rajasekhar VK, Sanghvi VR, Mavrakis KJ, Jiang M, Roderick JE, Van der Meulen J, et al: RNA G-quadruplexes cause eIF4A-dependent oncogene translation in cancer. Nature 513: 65-70, 2014.

15. Meijer HA, Kong YW, Lu WT, Wilczynska A, Spriggs RV, Robinson SW, Godfrey JD, Willis AE and Bushell M: Translational repression and eIF4A2 activity are critical for microRNA-mediated gene regulation. Science 340: 82-85, 2013.

16. Chan CC, Dostie J, Diem MD, Feng W, Mann M, Rappsilber J and Dreyfuss G: eIF4A3 is a novel component of the exon junction complex. RNA 10: 200-209, 2004.
17. Li Q, Imataka H, Morino S, Rogers GW Jr, Richter-Cook NJ, Merrick WC and Sonenberg N: Eukaryotic translation initiation factor 4AIII (eIF4AIII) is functionally distinct from eIF4AI and eIF4AII. Mol Cell Biol 19: 7336-7346, 1999.

18. Wilczynska A, Gillen SL, Schmidt T, Meijer HA, Jukes-Jones R, Langlais C, Kopra K, Lu WT, Godfrey JD, Hawley BR, et al: eIF4A2 drives repression of translation at initiation by Ccr4-Not through purine-rich motifs in the 5'UTR. Genome Biol 20: 262, 2019.

19. Ji P, Diederichs S, Wang W, Böing S, Metzger R, Schneider PM, Tidow N, Brandt B, Buerger H, Bulk E, et al: MALAT-1, a novel noncoding RNA, and thymosin beta4 predict metastasis and survival in early-stage non-small cell lung cancer. Oncogene 22: 8031-8041, 2003.

20. Liang S, Zhou Y, Chen Y, Ke G, Wen H and Wu X: Decreased expression of EIF4A1 after preoperative brachytherapy predicts better tumor-specific survival in cervical cancer. Int J Gynecol Cancer 24: 908-915, 2014

21. Lomnytska MI, Becker S, Gemoll T, Lundgren C, Habermann J, Olsson A, Bodin I, Engström U, Hellman U, Hellman K, et al: Impact of genomic stability on protein expression in endometrioid endometrial cancer. Br J Cancer 106: 1297-1305, 2012.

22. Modelska A, Turro E, Russell R, Beaton J, Sbarrato T, Spriggs K, Miller J, Gräf S, Provenzano E, Blows F, et al: The malignant phenotype in breast cancer is driven by eIF4A1-mediated changes in the translational landscape. Cell Death Dis 6: e1603, 2015.

23. Oblinger JL, Burns SS, Akhmametyeva EM, Huang J, Pan L, Ren Y, Shen R, Miles-Markley B, Moberly AC, Kinghorn $\mathrm{AD}$, et al: Components of the eIF4F complex are potential therapeutic targets for malignant peripheral nerve sheath tumors and vestibular schwannomas. Neuro Oncol 18: 1265-1277, 2016

24. Chan K, Robert F, Oertlin C, Kapeller-Libermann D, Avizonis D, Gutierrez J, Handly-Santana A, Doubrovin M, Park J, Schoepfer C, et al: eIF4A supports an oncogenic translation program in pancreatic ductal adenocarcinoma. Nat Commun 10: $5151,2019$.

25. Shaoyan X, Juanjuan Y, Yalan T, Ping H, Jianzhong L and Qinian W: Downregulation of EIF4A2 in non-small-cell lung cancer associates with poor prognosis. Clin Lung Cancer 14: 658-665, 2013.

26. Yan LX, Wu QN, Zhang Y, Li YY, Liao DZ, Hou JH, Fu J, Zeng MS, Yun JP, Wu QL, et al: Knockdown of miR-21 in human breast cancer cell lines inhibits proliferation, in vitro migration and in vivo tumor growth 13: R2, 2011.

27. Chen ZH, Qi JJ, Wu QN, Lu JH, Liu ZX, Wang Y, Hu PS, Li T, Lin JF, Wu XY, et al: Eukaryotic initiation factor 4A2 promotes experimental metastasis and oxaliplatin resistance in colorectal cancer. J Exp Clin Cancer Res 38: 196, 2019.

28. Rice TW, Kelsen DP and Blackstone EH: Esophagus and Esophagogastric Junction. In: AJCC Cancer Staging Manual, 8th edition. Amin MB, Edge SB and Greene FL (eds). New York, NY, Springer, 185-202, 2017.

29. Dancau AM, Simon R, Mirlacher M and Sauter G: Tissue microarrays. Methods Mol Biol 576: 49-60, 2010.

30. Hinnebusch AG: The scanning mechanism of eukaryotic translation initiation. Annu Rev Biochem 83: 779-812, 2014.

31. Meijer HA, Schmidt T, Gillen SL, Langlais C, Jukes-Jones R, de Moor CH, Cain K, Wilczynska A and Bushell M: DEAD-box helicase eIF4A2 inhibits CNOT7 deadenylation activity. Nucleic Acids Res 47: 8224-8238, 2019.

32. Cheng YF, Chen HS, Wu SC, Chen HC, Hung WH, Lin CH and Wang BY: Esophageal squamous cell carcinoma and prognosis in Taiwan. Cancer Med 7: 4193-4201, 2018

This work is licensed under a Creative Commons Attribution-NonCommercial-NoDerivatives 4.0 International (CC BY-NC-ND 4.0) License. 